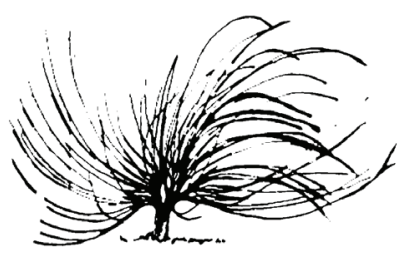

\title{
Uso de redes sociales digitales entre estudiantado universitario: Comunicación, socialización y colaboración
}

\section{Using digital social networks among college students: communication, socialization and collaboration}

\author{
Verónica Marini Munguíal \\ Nancy Jácome Ávila ${ }^{2}$ \\ Rocío López González ${ }^{3}$
}

\begin{abstract}
Resumen
Este artículo describe resultados sobre el uso de redes sociales digitales en actividades de comunicación, socialización y colaboración. Es parte de una investigación en proceso, cuyo propósito es analizar el uso que le da el estudiantado universitario a los dispositivos digitales portátiles (DDP) -laptop, tableta y teléfono inteligente-. Para recolectar la información se aplicó un cuestionario diseñado en el marco del proyecto Brecha digital entre estudiantes y profesores de la Universidad Veracruzana: Capital cultural; trayectorias escolares y desempeño académico; y grado de apropiación tecnológica. La muestra es de 329 estudiantes del Sistema de Enseñanza Abierta de la Universidad Veracruzana. Se destaca que los mensajes de texto SMS empiezan a ser sustituidos
\end{abstract}

1 Licenciada en Pedagogía por la Universidad Veracruzana. Maestra en Educación Virtual de la Universidad Veracruzana.

2 Académica del Programa de Investigación e Innovación en Educación Superior de la Universidad Veracruzana.

3 Doctora en Pedagogía por la Universidad Nacional Autónoma de México. Investigadora del Programa de Investigación e Innovación en Educación Superior de la Universidad Veracruzana. 
por aplicaciones como WhatsApp; redes sociales y correo electrónico son utilizados con mayor frecuencia para compartir y publicar información; se realizan pocas actividades de creación y colaboración con regularidad y Facebook es la red social más utilizada para comunicarse con docentes y pares. Se identificó cierta relación entre el género y algunas de las actividades que realizan en redes sociales, tales como establecer relaciones amorosas, usar mensajes de texto y comunicarse con compañeros y compañeras, y profesorado.

Palabras clave: Desarrollo tecnológico, dispositivos digitales portátiles, redes sociales digitales, educación abierta, estudiantado universitario.

\begin{abstract}
This paper describes the use of digital social networks for communication, socialization and collaboration as part of an ongoing investigation whose purpose is to analyze the use that university students make of portable digital devices (PDD) -Laptop, tablet and smartphone. To collect the data, a questionnaire was designed under the project "Digital divide between students and professors of the Universidad Veracruzana: Cultural Capital; school trajectories and academic performance; and degree of technological appropriation". The sample included 329 students of the open education system at the Universidad Veracruzana. It stresses that SMS text messages begin to be replaced by applications such as WhatsApp; social networks and email are frequently used to share and publish information; few are engaged in creation and collaboration with regularity and Facebook is the most used social network to communicate with teachers and peers. A certain relationship between gender and some of their activities in social networks, such as establishing relationships, using text messages and communicate with peers and teachers, was identified.
\end{abstract}

Keywords: technological development, Portable digital devices, Digital social networks, Open education, University students 


\section{Introducción}

$\mathrm{E}$ 1 desarrollo tecnológico en relación con la telefonía móvil y el cómputo ubicuo ${ }^{4}$ dio lugar a la creación de tecnologías como laptops, tabletas y teléfonos inteligentes, que en este artículo se identifican como dispositivos digitales portátiles (DDP). Esta situación da lugar a que en las últimas décadas se observe un incremento en la posesión y uso de dichos dispositivos en prácticamente todos los ámbitos de la vida cotidiana, principalmente en las generaciones más jóvenes (Cantillo, Roura y Sánchez, 2012; Fundación Telefónica y Fundación Encuentro, 2013).

El número de personas que hacen uso de celulares, tabletas o laptops de forma intensiva en diversos espacios de su vida tales como la calle, transporte público, oficinas, lugares de esparcimiento, escuelas, aumenta poco a poco. Esto, debido a que al reducirse el tamaño de los dispositivos su peso disminuye, y permite que se puedan llevar y usar en distintos lugares con mayor facilidad; asimismo, sus características de conexión a internet a través de redes $W i F i$, facilitan el acceso a búsqueda de información y redes sociales desde cualquier lugar donde se tenga el servicio, además, los costos de algunos dispositivos tienden a ser menores, al aumentar la oferta de equipos con una mayor variedad.

Dicho aumento en el uso de DDP favorece el acceso a información y redes sociales digitales en prácticamente cualquier momento y lugar que se desee, siempre que se tenga servicio de internet, lo que tiene como consecuencia una hiperconectividad (Fundación Telefónica y Fundación Encuentro, 2013), esto es, estar "conectados" prácticamente todo el tiempo. Situación que da lugar a nuevas formas de comunicación, interacción, socialización, entretenimiento e incluso de aprendizaje (Cobo, 2011), es decir, se abren nuevas formas de interconexión desde distintos lugares sin tener que estar de forma fija frente a una computadora de escritorio, más bien en movilidad a través de una laptop, tableta o teléfono inteligente (Urresti, 2011).

A partir del incremento en el acceso a los DDP y las transformaciones ocurridas en torno a este fenómeno, en el campo de la investigación educativa nacional se identifica un especial interés por desarrollar estudios

4 Tecnologías que permiten el acceso a información y servicios en el momento que se requiere sin importar tiempo y lugar. 
cuyo foco se centra en conocer (Javier, Romero y Ricoy, 2012), describir (Organista-Sandoval, Serrano-Santoyo, McAnally-Salas y Lavigne, 2013) y caracterizar (Cuen y Ramírez, 2013; Navarro, 2012) los usos y la apropiación de tecnologías de la información y comunicación (TIC) (Crovi, 2008), principalmente de teléfonos móviles (Carrera, Sapién y Piñón, 2013), en jóvenes dentro de diversos contextos, debido a que se identifican como el grupo poblacional que más los utilizan (Ortiz, 2011). De acuerdo con Castells, Fernández y Galperin (2011), ante el inminente aumento en el acceso a las tecnologías, últimamente en la telefonía móvil, ya no solo es relevante preguntarse sobre el acceso, sino también con qué frecuencia y para qué se usan, así como cuáles son las condiciones y los efectos que se generan con los DDP.

Cabe mencionar que existen diversos prejuicios en torno al tema, se suele pensar que los DDP son utilizados de manera igualitaria y generalizada por la mayoría de jóvenes; que estos grupos poseen habilidades para manejarlos sin dificultades; que solo los usan para comunicarse, entretenerse o producir contenidos superficiales; sin embargo, resultados de investigaciones muestran que esto no ocurre de forma general ni homogénea en todos los casos, ya que se identifican diferencias en relación con el contexto educativo, características culturales, socioeconómicas y demográficas de los estudiantes.

Cada grupo se apropia de la tecnología de acuerdo a sus prácticas y necesidades de comunicación, pero también de acuerdo a sus restricciones de recursos, y a su posicionamiento en redes sociales preexistentes, que a su vez se alteran a medida que estos actores adquieren mayor autonomía y mejor acceso a recursos disponibles en estas redes. (Castells et al., 2011, p. 344)

Si bien en el contexto mexicano se tienen cifras que dan cuenta del crecimiento en el acceso a DDP, y existen investigaciones en relación con el tema, es necesario realizar más estudios al respecto, debido a que este fenómeno se ha revisado principalmente en jóvenes estudiantes de instituciones públicas de modalidad educativa escolarizada y jóvenes trabajadores, y se dejan fuera a grupos de estudiantes de otras modalidades educativas, como es el caso de la abierta.

En este contexto, se consideró pertinente realizar un estudio sobre el uso de DDP en estudiantes del sistema de enseñanza abierta 
(SEA), región Xalapa, de la Universidad Veracruzana, debido a que se identificó que en dicha población aún no se desarrollan estudios que den cuenta de qué DDP poseen, con qué frecuencia los usan, qué habilidades tienen y qué actividades realizan en ellos.

Para este artículo, se propuso analizar el uso de redes sociales digitales para actividades de socialización, colaboración y comunicación que estudiantes del SEA realizan con sus DDP, a fin de conocer cómo se utilizan estas herramientas, ya que se trata de estudiantes con un rol distinto a los del sistema escolarizado, pues se basa en el estudio autónomo y la asistencia a sesiones presenciales los sábados, así como asesorías entre semana, esto implica poco tiempo para interactuar de manera presencial con compañeros, compañeras y profesorado.

\section{Referentes conceptuales}

Se entienden como dispositivos digitales portátiles aquellos aparatos electrónicos digitales fáciles de transportar por ser inalámbricos, con duración prolongada de la batería, peso y tamaño cómodos, con acceso a internet, procesador de memoria con características similares a una computadora, fáciles de manejar para realizar actividades de comunicación, entretenimiento, colaboración, búsqueda de información, almacenamiento de datos, grabación de video y audio, uso de redes sociales, ya sea en movimiento o en diferentes contextos (Aguilar, Ramírez y López, 2014; Fombona, Pascual y Madeira, 2012; Ramírez, 2014).

El uso se concibe como una intención definida a fin de obtener un beneficio relacionado con el aprovechamiento de las herramientas que los DDP ofrecen al realizar determinadas acciones de forma cotidiana (Crovi, 2009), que dependen del tipo de DDP y conexión a internet a que se tenga acceso; opiniones, habilidades y frecuencia con que se realizan distintas actividades.

Las redes sociales dentro de un entorno tecnológico se suelen identificar como digitales. Estas, acuerdo con Constante (2013), son:

Un portal de Internet que permite a las personas construir un perfil público o semipúblico dentro de los límites de la plataforma que ofrece los servicios que suelen ser muy variados y generalmente están constituidas por un grupo de personas ligado por intereses comunes, abierto a compartir pensamientos, 
pero también pedazos de la propia vida: desde enlaces a sitios que consideran interesantes hasta las fotografías o los propios videos personales (p. 28).

Es decir, las redes sociales digitales son aquellas interacciones que se establecen entre dos o más personas a través de plataformas alojadas en internet, con distintos propósitos, ya sea personales, sociales, laborales e incluso escolares, a través de procesos de comunicación, socialización y colaboración.

Ramírez y Casillas (2014) proponen 10 saberes mínimos digitales necesarios para poder interactuar en contextos digitales, dos de estos saberes son:

a. Comunicarse en entornos digitales, que hace referencia a:

"Conocimientos y habilidades para transmitir información (voz, mensajes de texto, fotos o videollamadas) a uno o más destinatarios; o recibirla de uno o más remitentes de manera sincrónica llamada, videoconferencia o chat) o asincrónica (correo electrónico, mensajes de texto, correo de voz)" (Ramírez y Casillas, 2014, p. 7).

b. Socializar y colaborar en entornos digitales, que consiste en:

"Conocimientos y habilidades orientadas a la difusión de información (blogs, microblogs), interacción social (redes sociales como Facebook, Twitter, Instagram), presencia en web (indicar "me gusta", hacer comentarios en servidores de medios o blogs, marcado social) y al trabajo grupal mediado por web (plataformas de colaboración como google docs o entornos virtuales de aprendizaje como Moodle y Eminus)" (Ramírez y Casillas, 2014, p. 8).

Con base en lo antes mencionado, para conocer el uso de redes sociales digitales se tomarán en cuenta tres categorías: a) actividades, b) comunicación, c) socialización y colaboración.

\section{Método y participantes}

Este trabajo fue de tipo cuantitativo, no experimental, transversal descriptivo. Se aplicó un cuestionario a un grupo de estudiantes del SEA, Xalapa, que ha cursado un año o más de su licenciatura, inscrito 
en el periodo agosto 2014 - enero 2015. Se calculó una muestra de 329 estudiantes (correspondiente al 19\% de la población ${ }^{5}$ ), los que se seleccionaron a través de un muestreo no probabilístico intencionado, a fin de garantizar la elección de integrantes de las cuatro licenciaturas. El total de la muestra se distribuyó de manera proporcional, quedó como sigue: administración 73, contaduría 72, derecho 90 y pedagogía 94 .

El instrumento aplicado se diseñó en el marco del proyecto Brecha digital entre estudiantes y profesores de la Universidad Veracruzana: Capital cultural; trayectorias escolares y desempeño académico; y grado de apropiación tecnológica, por docentes y estudiantes de la línea de investigación Políticas en educación superior del Instituto de Investigaciones en Educación, UV. Tiene 42 ítems, está estructurado por preguntas cerradas con escalas Likert, dicotómicas y algunas abiertas. Está dividido en 11 secciones, para este artículo solo se presentan los resultados de la sección: comunicación, socialización y colaboración, con el propósito de dar cuenta de qué actividades realizan y con qué frecuencia usan las redes sociales el estudiantado universitario en sus DDP.

Aunque el cuestionario se encuentra en versión digital e impresa, ${ }^{6}$ se optó por aplicar esta segunda opción, debido a que el estudiantado del SEA no cuenta con centro de cómputo propio, pues las computadoras a las que tiene acceso - para clases de computación básica- son prestadas por las facultades del sistema escolarizado. La aplicación se realizó en diversos salones de las cuatro licenciaturas en el turno matutino, se eligieron grupos con estudiantes de tercer semestre en adelante, debido a que se pretendió recuperar sus opiniones a partir del nivel de avance dentro de la licenciatura.

Del grupo de participantes más de la mitad son mujeres $(60.5 \%)$ en contraste con el $39.5 \%$ de hombres, característica que corresponde con el total de la población, ya que en la distribución de la matrícula estudiantil del SEA predominan las mujeres. Al interior de las licenciaturas se observó que solo en administración se tuvo una

5 La población total de estudiantes que han cursado un año o más de su licenciatura inscritos en el periodo agosto 2014 - enero 2015 en la región de Xalapa estuvo conformada por 1,717 estudiantes.

6 Se puede acceder a las dos versiones del cuestionario en la siguiente liga: http://www.uv.mx/ blogs/brechadigital/informacion/ 
mayor participación de hombres (53.4\%), pues en el resto la presencia femenina se encuentra arriba del $50 \%$.

En cuanto a la edad, estudiantes que participaron en la investigación tienen en promedio 25 años, esto muestra que los resultados corresponden a personas relativamente jóvenes. El rango de edad que concentra parte de la muestra es de 22 a 25 (36.5\%), seguido por el de 18 a 21 (28.6\%), es decir, el 65\% tiene entre 18 y 25 años, dato que es importante resaltar debido a que el SEA, en sus inicios se caracterizaba por atender a personas adultas, lo cual refleja que la población que atiende este sistema ha cambiado a lo largo del tiempo, pues en este caso las personas mayores a 30 años solo representan un $17.9 \%$.

Asimismo, cabe agregar que actualmente se cuenta con estudiantes jóvenes, su principal actividad es estudiar; estudiantes trabajadores, quienes generalmente cuentan con carreras previas o son semiprofesionistas, cuyo ingreso al sistema les permite mejorar su situación laboral, los cuales eran la población original del SEA y, por último, están estudiantes con una carrera universitaria previa o en curso (Alarcón, 2012; Tepetla, 2008).

Para el procesamiento de la información se elaboró una base de datos en el programa estadístico SPSS (Statistical Package for the Social Sciences), versión 22 para Windows. El análisis que se realizó fue exploratorio univariante, mediante la descripción de cada una de las preguntas de la sección de comunicación, socialización y colaboración, a través de tablas de frecuencias, así como un análisis de relación entre género y cada uno de los ítems, mediante tablas de contingencia con el estadístico Chi-cuadrado.

\section{Resultados}

De acuerdo con los datos, la mayoría de estudiantes del SEA señaló usar redes sociales. A continuación, se presentan las actividades que realizan a través de ellas y la frecuencia con que lo hacen. Tal como se muestra en la tabla 1, la mayoría de las actividades enlistadas son realizadas de manera frecuente. 
Tabla 1

Frecuencia de actividades en redes sociales digitales

\begin{tabular}{lcccccc}
\hline \multicolumn{1}{c}{ Actividades } & Siempre & $\begin{array}{c}\text { Frecuente- } \\
\text { mente }\end{array}$ & $\begin{array}{c}\text { Algunas } \\
\text { veces }\end{array}$ & $\begin{array}{c}\text { Casi } \\
\text { nunca }\end{array}$ & Nunca & $\begin{array}{c}\text { No } \\
\text { contestó }\end{array}$ \\
\hline Jugar & $1.8 \%$ & $9.7 \%$ & $20.4 \%$ & $28.0 \%$ & $39.8 \%$ & $0.3 \%$ \\
Contactar amigos/as & $18.5 \%$ & $45.0 \%$ & $26.1 \%$ & $7.6 \%$ & $2.4 \%$ & $0.3 \%$ \\
Contactar compañeros/as & $17.3 \%$ & $42.2 \%$ & $33.1 \%$ & $5.5 \%$ & $1.8 \%$ & 0 \\
Contactar familiares & $17.9 \%$ & $37.1 \%$ & $29.2 \%$ & $10.6 \%$ & $4.9 \%$ & $0.3 \%$ \\
Relaciones amorosas & $6.7 \%$ & $13.4 \%$ & $18.8 \%$ & $20.7 \%$ & $39.5 \%$ & $0.9 \%$ \\
Leer noticias & $24.9 \%$ & $41.3 \%$ & $24.6 \%$ & $5.5 \%$ & $3.6 \%$ & 0 \\
$\begin{array}{l}\text { Participar en actividades } \\
\text { en grupo }\end{array}$ & $9.1 \%$ & $21.9 \%$ & $37.4 \%$ & $20.7 \%$ & $10.9 \%$ & 0 \\
\hline
\end{tabular}

Nota: Elaboración propia.

Cabe resaltar que jugar y establecer relaciones amorosas a través de redes sociales son actividades que se realizan con poca frecuencia, pues el 28\% y $39.9 \%$ indicaron jugar "Casi nunca" y "Nunca", respectivamente; de igual forma, el 20.7\% "Casi nunca" establece relaciones amorosas y $39.5 \%$ señaló "Nunca" hacerlo. En contraste con aquellas que realizan "Frecuentemente", tales como contactar amigos (45\%), compañeros (42.2\%), familiares (37.1\%) y leer noticias (41.3\%).

Se aplicó la prueba de independencia de Chi cuadrada para identificar la existencia de relación entre el género y las diversas actividades que los sujetos participantes llevan a cabo en redes sociales, a partir de los resultados se identificó que establecer relaciones amorosas $\left(X_{g l .4}^{2}=11.53 ; \mathrm{p}=.021\right)$ en redes sociales está relacionado con el género.

De igual forma, se presentan los medios que los estudiantes utilizan para comunicarse y la frecuencia con que lo realizan. La principal forma de comunicación que la mayoría utiliza "Siempre" es el chat en dispositivo móvil, tal como WhatsApp o Telegram (60.2\%), 
seguida de las redes sociales en dispositivo móvil (41\%). El correo electrónico en computadora (37.1\%), el chat en computadora (33.7\%) y las redes sociales en computadora (33.1\%) son los medios utilizados con "Frecuencia" entre la población. Por otra parte, llama la atención que la videollamada tanto en computadora (29.2\%) como en dispositivo móvil (41.3\%) "Nunca" la utilizan (ver tabla 2).

Tabla 2

Frecuencia de uso de herramientas para comunicación

\begin{tabular}{|c|c|c|c|c|c|c|}
\hline Herramientas & Siempre & Frecuente & Algunas & & Nunca & No \\
\hline & & & veces & nunca & vinta & contestó \\
\hline Chat en computadora & $17.9 \%$ & $33.7 \%$ & $28.9 \%$ & $15.5 \%$ & $4.0 \%$ & 0 \\
\hline $\begin{array}{l}\text { Chat en dispositivo móvil } \\
\text { (WhatsApp, Telegram) }\end{array}$ & $60.2 \%$ & $27.4 \%$ & $6.7 \%$ & $3.0 \%$ & $2.7 \%$ & 0 \\
\hline $\begin{array}{l}\text { Correo electrónico en } \\
\text { computadora }\end{array}$ & $36.2 \%$ & $37.1 \%$ & $20.1 \%$ & $5.8 \%$ & $0.9 \%$ & 0 \\
\hline $\begin{array}{l}\text { Correo electrónico en } \\
\text { dispositivo móvil }\end{array}$ & $28.0 \%$ & $24.3 \%$ & $21.6 \%$ & $17.0 \%$ & $8.8 \%$ & $0.3 \%$ \\
\hline $\begin{array}{l}\text { Redes sociales en } \\
\text { computadora }\end{array}$ & $29.2 \%$ & $33.1 \%$ & $25.8 \%$ & $10.9 \%$ & $0.9 \%$ & 0 \\
\hline $\begin{array}{l}\text { Redes sociales en } \\
\text { dispositivo móvil }\end{array}$ & $41.0 \%$ & $29.8 \%$ & $15.2 \%$ & $9.1 \%$ & $4.6 \%$ & $0.3 \%$ \\
\hline $\begin{array}{l}\text { Plataformas de } \\
\text { aprendizaje distribuido } \\
\text { (EMINUS, Moodle) en } \\
\text { computadora }\end{array}$ & $11.2 \%$ & $26.4 \%$ & $31.0 \%$ & $20.1 \%$ & $10.0 \%$ & $1.2 \%$ \\
\hline $\begin{array}{l}\text { Plataformas de } \\
\text { aprendizaje distribuido } \\
\text { (EMINUS, Moodle) en } \\
\text { móvil }\end{array}$ & $7.0 \%$ & $12.8 \%$ & $23.7 \%$ & $35.0 \%$ & $20.4 \%$ & $1.2 \%$ \\
\hline $\begin{array}{l}\text { Videollamada en } \\
\text { computadora }\end{array}$ & $6.4 \%$ & $9.4 \%$ & $27.7 \%$ & $26.7 \%$ & $29.2 \%$ & $0.6 \%$ \\
\hline $\begin{array}{l}\text { Videollamada en } \\
\text { dispositivo móvil }\end{array}$ & $6.1 \%$ & $8.8 \%$ & $15.5 \%$ & $28.0 \%$ & $41.3 \%$ & $0.3 \%$ \\
\hline Mensaje de texto (SMS) & $21.0 \%$ & $23.4 \%$ & $31.9 \%$ & $18.8 \%$ & $4.9 \%$ & 0 \\
\hline
\end{tabular}

Nota: Elaboración propia. 
Otro dato que es importante resaltar es que si se suman las columnas "Siempre" y "Frecuentemente" para mensajes de texto (44.4\%), se observa que el porcentaje de estudiantes que los utilizan es menor al de quienes se comunican por chat en el dispositivo móvil (87.2\%), lo cual tiene sentido, si se toma en cuenta que aunque los dos son sistemas de comunicación similares, el segundo es sin costo, si se tiene acceso a un red wi fi.

Respecto a la prueba de independencia Chi cuadrada que se realizó para conocer la relación entre el género y cada una de las herramientas que el estudiantado utiliza con fines de comunicación, se encontró con los valores obtenidos que existe relación entre el género y el uso de mensajes de texto SMS $\left(X^{2}{ }_{g l .4}=11.20 ; \mathrm{p}=.024\right)$.

En congruencia con los resultados de la sección anterior, se puede apreciar en la tabla 3 que al sumar las columnas "Siempre" y "Frecuentemente" las redes sociales (72.3\%) son el medio más utilizado de forma continua para publicar y compartir información, seguido del correo electrónico (70.3\%), al ser estas las principales herramientas de comunicación utilizadas por la comunidad estudiantil. En contraste con las plataformas de aprendizaje distribuido, como Moodle, un 31.9\% señaló "Casi nunca" utilizarlas, mientras que en las páginas personales más de una cuarta parte dijo "Nunca" publicar ni compartir información.

\section{Tabla 3}

Frecuencia en compartir y publicar información

\begin{tabular}{lccccc}
\hline \multicolumn{1}{c}{ Medios } & \multirow{2}{*}{ Siempre } & Frecuente- Algunas & Casi & \multirow{2}{*}{ Nunca } \\
& & mente & veces & nunca & \\
\hline Correo electrónico & $35.0 \%$ & $35.3 \%$ & $19.8 \%$ & $7.9 \%$ & $2.1 \%$ \\
Redes sociales & $32.2 \%$ & $40.1 \%$ & $18.5 \%$ & $8.2 \%$ & $0.9 \%$ \\
Blogs y páginas personales & $4.9 \%$ & $11.6 \%$ & $20.4 \%$ & $29.2 \%$ & $34.0 \%$ \\
Plataformas de aprendizaje & $5.2 \%$ & $12.5 \%$ & $24.3 \%$ & $31.9 \%$ & $26.1 \%$ \\
distribuido (como EMINUS o & & & & & \\
Moodle) & & & & & \\
\hline
\end{tabular}

Nota: Elaboración propia.

Al analizar la relación entre el género y los medios que los sujetos encuestados utilizan para compartir información, los resultados (correo electrónico $X_{\text {gl. }}^{2}=1.54 ; \mathrm{p}=.818$; redes sociales $X_{g l .4}^{2}=7.15 ; \mathrm{p}=$ 
.128; blogs y páginas personales $X_{g l .4}^{2}=2.78 ; \mathrm{p}=.595$; plataformas de aprendizaje distribuido $X_{\text {gl. }}^{2}=2.64 ; \mathrm{p}=.620$;) conducen a constatar que no existe relación entre el género y los cuatro medios utilizados para compartir información, es decir, el uso de dichos medios no depende del género.

Otro aspecto que se buscó conocer fue si el estudiantado realiza actividades de creación, colaboración y socialización, así como conocer las formas en que se comunica con compañeros, compañeras y docentes, todo ello con fines académicos y no académicos, además de la frecuencia con que lo realizan. Los resultados que se presentan en la tabla 4 dan cuenta de que para fines académicos la mayoría lleva a cabo actividades de creación y colaboración con escasa frecuencia, ejemplo de esto es que el $68.4 \%$ indicó "Nunca" administrar un blog personal, un porcentaje más alto señaló lo mismo sobre la administración de algún canal de videos (78.4\%) y solo el 3.6\% dijo "Siempre" crear y compartir documentos en línea a través de google docs.

\section{Tabla 4}

Frecuencia de actividades de comunicación, socialización y colaboración con fines académicos

\begin{tabular}{|c|c|c|c|c|c|c|}
\hline Acciones & Siempre & $\begin{array}{c}\text { Frecuente- } \\
\text { mente }\end{array}$ & $\begin{array}{c}\text { Algunas } \\
\text { veces }\end{array}$ & $\begin{array}{c}\text { Casi } \\
\text { nunca }\end{array}$ & Nunca & $\begin{array}{c}\text { No } \\
\text { contestó }\end{array}$ \\
\hline $\begin{array}{l}\text { Administro uno o } \\
\text { varios blogs personales }\end{array}$ & $1.2 \%$ & $3.3 \%$ & $10.9 \%$ & $13.1 \%$ & $68.4 \%$ & $3.0 \%$ \\
\hline $\begin{array}{l}\text { Consulto bases de } \\
\text { datos y repositorios } \\
\text { institucionales }\end{array}$ & $4.6 \%$ & $15.5 \%$ & $32.5 \%$ & $19.1 \%$ & $26.7 \%$ & $1.5 \%$ \\
\hline $\begin{array}{l}\text { Administro un canal } \\
\text { de videos (en YouTube } \\
\text { o en plataformas } \\
\text { similares) }\end{array}$ & $1.8 \%$ & $3.6 \%$ & $5.8 \%$ & $8.2 \%$ & $78.4 \%$ & $2.1 \%$ \\
\hline $\begin{array}{l}\text { Creo y comparto } \\
\text { documentos en línea } \\
\text { en herramientas como } \\
\text { Google Docs }\end{array}$ & $3.6 \%$ & $6.7 \%$ & $14.3 \%$ & $12.8 \%$ & $60.5 \%$ & $2.1 \%$ \\
\hline Uso Twitter & $1.8 \%$ & $5.5 \%$ & $10.3 \%$ & $10.3 \%$ & $69.0 \%$ & $3.0 \%$ \\
\hline Uso Facebook & $17.9 \%$ & $32.2 \%$ & $29.2 \%$ & $10.3 \%$ & $9.4 \%$ & $0.9 \%$ \\
\hline
\end{tabular}




\begin{tabular}{lllllll} 
Uso Instagram & $0.9 \%$ & $2.7 \%$ & $3.6 \%$ & $10.9 \%$ & $79.0 \%$ & $2.7 \%$ \\
\hline $\begin{array}{l}\text { Me comunico con mis } \\
\text { profesores/as mediante }\end{array}$ & $7.9 \%$ & $17.3 \%$ & $35.3 \%$ & $18.2 \%$ & $19.5 \%$ & $1.8 \%$ \\
$\begin{array}{l}\text { redes sociales } \\
\text { Me comunico con mis } \\
\text { compañeros/as de la }\end{array}$ & & & & & \\
$\begin{array}{l}\text { Universidad por medio } \\
\text { de redes sociales }\end{array}$ & & & & & & \\
\hline Nota: Elaboración propia. & & & & & & \\
\end{tabular}

En relación con las actividades de socialización con fines académicos, al sumar las columnas de "Siempre" y "Frecuentemente", se identificó que Facebook (50.1\%) es la plataforma que se utiliza con mayor frecuencia para ello, en contraste con más de la mitad que señalaron "Nunca" hacer uso de Instagram (79\%) y Twitter (69\%). La comunicación entre estudiantes y sus docentes a través de redes sociales se lleva a cabo con poca regularidad, pues solo $7.9 \%$ dijo realizarlo "Siempre", un 35.3\% señaló comunicarse "Algunas veces" y el 19.5\% "Nunca" lo hace. Mientras que, la comunicación a través de este medio se da con mayor frecuencia entre pares de la clase, el $36.2 \%$ se comunica "Siempre" en contraste con el 3\% que dijo "Nunca" realizarlo.

En el caso de la relación entre el género y las actividades que el estudiantado realiza con fines académicos en redes sociales, los resultados de la prueba indican que en los ítems "Me comunico con mis profesores" $\left(X_{g l .4}^{2}=14.50 ; \mathrm{p}=.006\right)$ y "Me comunico con mis compañeros" $\left(X_{\text {gl. }}^{2} \stackrel{\text { gl.4 }}{=} 10.07 ; \mathrm{p}=.039\right)$, existe relación con el género, por lo que es posible afirmar que la forma en que el estudiantado participante se comunica con sus docentes y sus pares está relacionada con ser hombre o mujer.

Por otra parte, para fines no académicos se observa que en la opción "Consultó bases de datos y repositorios institucionales" los porcentajes de "Nunca" se incrementan de $26.7 \%$ (académico) a un $50.5 \%$ (no académico), en tanto que, administrar un canal de videos en YouTube disminuye de un 78.4\% (académico) a un 74.2\% (no académico). Una situación similar a este segundo caso ocurre con las plataformas de socialización, puesto que los porcentajes de "Nunca" en 
el uso de Twitter, Facebook e Instagram disminuyen y aumentan los de "Siempre", esto se puede apreciar en la tabla 5:

\section{Tabla 5}

Frecuencia de actividades de comunicación, socialización y colaboración con fines no académicos

\begin{tabular}{|c|c|c|c|c|c|c|}
\hline Acciones & Siempre & $\begin{array}{c}\text { Frecuente- } \\
\text { mente }\end{array}$ & $\begin{array}{c}\text { Algunas } \\
\text { veces }\end{array}$ & $\begin{array}{c}\text { Casi } \\
\text { nunca }\end{array}$ & Nunca & $\begin{array}{c}\text { No } \\
\text { contestó }\end{array}$ \\
\hline $\begin{array}{l}\text { Administro uno o varios } \\
\text { blogs personales }\end{array}$ & $2.7 \%$ & $5.8 \%$ & $8.8 \%$ & $14.0 \%$ & $66.0 \%$ & $2.7 \%$ \\
\hline $\begin{array}{l}\text { Consulto bases de } \\
\text { datos y repositorios } \\
\text { institucionales }\end{array}$ & $1.5 \%$ & $6.7 \%$ & $15.5 \%$ & $21.3 \%$ & $50.5 \%$ & $4.6 \%$ \\
\hline $\begin{array}{l}\text { Administro un canal } \\
\text { de videos (en YouTube } \\
\text { o en plataformas } \\
\text { similares) }\end{array}$ & $2.1 \%$ & $4.9 \%$ & $7.9 \%$ & $7.9 \%$ & $74.2 \%$ & $3.0 \%$ \\
\hline $\begin{array}{l}\text { Creo y comparto } \\
\text { documentos en línea } \\
\text { en herramientas como }\end{array}$ & $1.2 \%$ & $3.3 \%$ & $11.6 \%$ & $11.6 \%$ & $69.3 \%$ & $3.0 \%$ \\
\hline Google Docs & & & & & & \\
\hline Uso Twitter & $7.6 \%$ & $10.0 \%$ & $14.0 \%$ & $9.1 \%$ & $56.5 \%$ & $2.7 \%$ \\
\hline Uso Facebook & $34.3 \%$ & $38.6 \%$ & $14.9 \%$ & $5.5 \%$ & $2.7 \%$ & $4.0 \%$ \\
\hline Uso Instagram & $11.6 \%$ & $9.4 \%$ & $6.4 \%$ & $7.0 \%$ & $62.6 \%$ & $3.0 \%$ \\
\hline $\begin{array}{l}\text { Me comunico con mis } \\
\text { profesores/as mediante } \\
\text { redes sociales }\end{array}$ & $5.2 \%$ & $8.8 \%$ & $16.1 \%$ & $14.0 \%$ & $51.1 \%$ & $4.9 \%$ \\
\hline $\begin{array}{l}\text { Me comunico con mis } \\
\text { compañeros/as de la } \\
\text { Universidad por medio } \\
\text { de redes sociales }\end{array}$ & $38.0 \%$ & $31.9 \%$ & $13.7 \%$ & $5.5 \%$ & $7.3 \%$ & $3.6 \%$ \\
\hline
\end{tabular}

Nota: Elaboración propia.

Respecto a la comunicación a través de redes sociales, la que se da entre profesorado y estudiantado disminuye considerablemente, pues el porcentaje de "Nunca" aumenta de 19.5\% (académico) a 51.1\% (no académico), mientras que la que se realiza entre compañeros y 
compañeras aumenta, los porcentajes de "Siempre" se incrementan de $36.2 \%$ (académico) a $38 \%$ (no académico).

Los resultados al aplicar la prueba Chi cuadrada, para analizar la relación entre el género y las actividades que el estudiantado realiza en redes sociales con fines no académicos, permiten observar que el uso de twitter $\left(X_{g l .4}^{2}=10.07 ; \mathrm{p}=.039\right)$ se relaciona con ser hombre o mujer.

\section{Consideraciones finales}

Este estudio sirvió como un primer acercamiento al tipo de redes sociales digitales que utilizan estudiantes del SEA, a las actividades que realizan en ellas y la frecuencia con que las usan. Los datos muestran que, de manera general, las tres herramientas que utilizan con mayor frecuencia para fines de comunicación son el chat en WhatsApp, redes sociales y correo electrónico.

Asimismo, se logró observar cómo el uso del chat a través de plataformas como WhatsApp y de redes sociales se llevan a cabo con mayor frecuencia a través de los dispositivos digitales portátiles que en una computadora de escritorio, esto gracias a las características de portabilidad y movilidad que ofrecen dichos dispositivos, lo cual permite que estas herramientas puedan ser utilizadas en cualquier lugar y momento que se desee, toda vez que se tenga acceso a internet.

Otro aspecto que se logró identificar es que los mensajes de texto SMS empiezan a ser sustituidos por nuevas herramientas de comunicación como WhatsApp, que tienen características similares a los primeros y no tienen costo, si se está conectado a internet a través de una red $W i F i$, cualidad que probablemente sea una de las principales razones por la que se elija esta herramienta en lugar de los mensajes. Por otra parte, las videollamadas son otro tipo de herramienta que no tiene costo, sin embargo, los participantes señalaron utilizarlas con poca frecuencia, lo cual refleja que el uso de dichas herramientas de comunicación no se da de manera homogénea ni de forma permanente. Esto invita a seguir explorando por qué se prefiere la comunicación a través de mensajes escritos en lugar de llamadas con video, o para qué tipo de situaciones se usan unas y no otras, o si la falta de uso se debe a desconocimiento de las herramientas, formas de uso o por desinterés.

Los medios que usan con mayor frecuencia para comunicarse son también para compartir y publicar información, así las redes sociales y 
el correo electrónico poseen altos porcentajes en los rubros que tienen relación con un mayor uso. Pese a estos datos, se logró identificar que solo un número pequeño señaló realizar actividades de creación y colaboración independientemente si son con fines académicos o no académicos. Estos resultados incitan a reflexionar y seguir investigando sobre las formas de producción y consumo de contenido e información en redes sociales digitales.

En cuanto al uso de redes sociales para fines académicos y no académicos, Facebook resultó ser la más utilizada, la cual sirve al estudiantado para comunicarse "Algunas veces" con su profesorado para fines académicos y de manera regular con sus compañeros y compañeras para ambos fines: académicos y no académicos. Los resultados de la prueba Chi cuadrada permitieron identificar algunas relaciones entre el género y la comunicación con docentes y pares en el ámbito académico, lo cual indica que la realización de este tipo de actividades depende del género, por ello es necesario realizar estudios de corte cualitativo que permitan explicar este tipo de relación.

También, se descubrió que actividades como jugar y establecer relaciones amorosas son realizadas con poca frecuencia a través de redes sociales en este grupo de la población, más bien las utilizan principalmente para estar en contacto con sus amistades, compañeros, compañeras y familiares, así como para leer noticias y participar en actividades en grupo. En suma, los resultados permitieron observar que el uso de redes sociales entre el estudiantado participante no es igual ni se da con la misma frecuencia, aunado a ello se identificó cierta relación entre el género y algunas actividades, por lo cual es importante realizar otro tipo de estudios que permitan analizar con más detalle estas situaciones.

\section{Referencias}

Aguilar, J. L., Ramírez, A., y López, R. (2014). Literacidad digital académica de los estudiantes universitarios: Un estudio de caso. Revista Electrónica de Investigación y Docencia (REID), 11, 123146. Recuperado de http://revistaselectronicas.ujaen.es/index. $\mathrm{php} / \mathrm{reid} / \mathrm{article} / \mathrm{view} / 1257$

Alarcón, E. A. (2012). La experiencia escolar en el Sistema de Enseñanza Abierta de la Universidad Veracruzana (Maestría). 
Universidad Veracruzana, Xalapa de Enríquez, Veracruz, México. Recuperado de http://www.uv.mx/mie/files/2012/10/Tesis Exciani.pdf

Cantillo, C., Roura, M., y Sánchez, A. (2012). Tendencias actuales en el uso de dispositivos móviles en educación. La Educación Digital Magazine, 147, 1-21.

Carrera, M., Sapién, A. L., y Piñón, L. C. (2013). Uso del teléfono inteligente con fines académicos: Caso de estudio: FCA de la $U A C H$. Presentado en el XVI Congreso Internacional sobre Innovaciones en Docencia e Investigación en Ciencias Económico Administrativas. Recuperado de http://www.fca.uach.mx/apcam/2013/11/22/p64_UACH.pdf

Castells, M., Fernández, M., y Galperin, H. (2011). Síntesis de resultados y conclusiones. En M. Fernández, H. Galperin y M. Castells, Comunicación móvil y desarrollo económico y social en América Latina (pp. 319-350). España: Ariel, Fundación Telefónica y Planeta. Recuperado de http://www.fundacion.telefonica.com/ es/arte cultura/publicaciones/detalle/95

Cobo, C. (2011). Casos y experiencias para aprender. En C. Cobo y J. W. Moravec, Aprendizaje invisible: Hacia una nueva ecología de la educación (pp. 107-140). Barcelona: Col.lecció Transmedia XXI. Laboratori de Mitjans Interactius. Publicacions i Edicions de la Universitat de Barcelona. Recuperado de www.aprendizajeinvisible.com

Constante. A. (Coord.). (2013). Las redes sociales. Una manera de pensar el mundo. México:Autor.

Crovi, D. (2008). Diagnóstico acerca del acceso, uso y apropiación de las TIC en la UNAM. Anuario Ininco, 20(1), 79-95.

Crovi, D. (2009). Acceso, uso y apropiación de las TIC en comunidades académicas. Diagnóstico en la UNAM. México: Universidad Nacional Autónoma de México y Plaza y Valdés.

Cuen. C., y Ramírez, J. L. (2013). TIC: Usos y efectos en el proceso de enseñanza-aprendizaje de una licenciatura en ciencias de la comunicación. Presentado en el XII Congreso Nacional de Investigación Educativa, Guanajuato, Guanajuato, México.

Fombona, L., Pascual, M. A., y Madeira, A. F. (2012). Realidad aumentada, una evolución de las aplicaciones de los dispositivos móviles. Pixel-Bit: Revista de Medios Y Educación, 41, 197-210. 
Fundación Telefónica, y Fundación Encuentro. (2013). Un nuevo escenario. En D. Reig y L. F. Vílchez, Los jóvenes en la era de la hiperconectividad: Tendencias, claves y miradas (pp. 9-19). España: Fundación Telefónica y Fundación Encuentro. Recuperado de http://www.fundacion.telefonica.com/es/arte_cultura/ publicaciones/detalle/182

INEGI. (2014). Usuarios de internet por equipo principal utilizado para conectarse a internet, 2010-2013. Recuperado de http://www3. inegi.org.mx/sistemas/temas/default.aspx? $=\mathrm{s}=\mathrm{est} \& \mathrm{c}=19007$

Javier, A. E., Romero, L. C. y Ricoy, C. M. (2012). El uso e impacto de las TIC en los estudiantes del nivel superior: Un estudio en las carreras de Derecho y Sociología de la UJAT. Perspectivas Docentes, 50, 5-11.

Navarro, M. (2012). El m-learning y los usos de tablets y celulares en el aula de clase, ¿distractores o dinamizadores del aprendizaje? En M. Navarro y R. Edel, Las TIC en la educación, un abordaje integrador (pp. 111-139). México: Red Durango de Investigadores Educativos. Recuperado de http://redie.mx/librosyrevistas/ libros/coleccionlibro4.pdf

Organista, J., Serrano, A., McAnally, L., y Gilles, L. (2013). Apropiación y usos educativos del celular por estudiantes y docentes universitarios. Revista Electrónica de Investigación Educativa, 15(3), 139-156.

Ortiz, G. (2011). Jóvenes, computadoras e internet: Usos, apropiaciones y sentidos. El caso de las y los jóvenes estudiantes del Tec y de la UIN (Tesis de doctorado). Universidad Autónoma Metropolitana Unidad Iztapalapa, México. Recuperado de http://tesiuami.izt.uam.mx/uam/aspuam/presentatesis.php?recno=16291\&docs=UAMI16291.pdf

Ramírez, A., y Casillas, M. A. (2014). Saberes digitales: Hojas de trabajo. Recuperado de http://www.uv.mx/blogs/ brechadigital/2014/08/24/hojas_saberes_digitales/

Ramírez, M. S. (2014). Recursos tecnológicos para el aprendizaje móvil (Mlearning) y su relación con los ambientes de educación a distancia: Implementaciones e investigaciones. RIED. Revista Iberoamericana de Educación a Distancia, 12(2).

Tepetla, J. (2008). Hacia un proyecto educativo alternativo en la carrera de Sociología-SEA. En J. Tepetla, G. Díaz y N. J. León, 
Sociología, educación y nuevas tecnologías ( $1^{\mathrm{a}}$ ed., pp. 35-56). Xalapa, México: Universidad Veracruzana.

Urresti, M. (2011). Las cuatro pantallas y las generaciones jóvenes. En A. Artopoulos (Coord.), La sociedad de las cuatro pantallas. Una mirada latinoamericana (pp. 3-29). España: Ariel, Fundación Telefónica y Planeta. Recuperado de http://www.fundacion. telefonica.com/es/arte_cultura/publicaciones/detalle/169 